OPEN ACCESS

Monitoring and Modeling the Variation of Electrochemical Current Induced by Dynamic Strain at Gold Surfaces

To cite this article: Qibo Deng and Aiting Yuan 2019 J. Electrochem. Soc. 166 H480

View the article online for updates and enhancements.

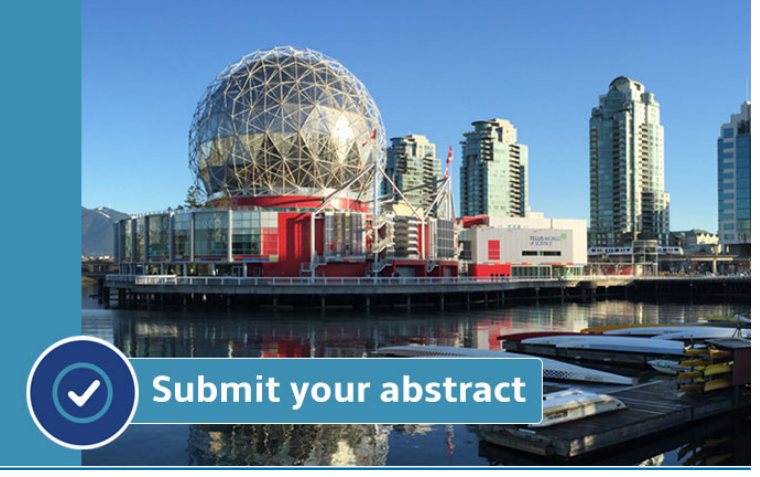




\title{
Monitoring and Modeling the Variation of Electrochemical Current Induced by Dynamic Strain at Gold Surfaces
}

\author{
Qibo Deng ${ }^{1,2,3, *, z}$ and Aiting Yuan ${ }^{1}$ \\ ${ }^{I}$ Tianjin Key Laboratory of Advanced Functional Porous Materials, Institute for New Energy Materials and \\ Low-Carbon Technologies, School of Materials Science and Engineering, Tianjin University of Technology, Tianjin \\ 300384, China \\ ${ }^{2}$ Institute of Material Physics and Technology, Hamburg University of Technology, Hamburg 21073, Germany \\ ${ }^{3}$ Key Laboratory of Advanced Energy Materials Chemistry (Ministry of Education), College of Chemistry, Nankai \\ University, Tianjin 300071, China
}

\begin{abstract}
This study investigates the electrochemical charging and discharging processes at a strained gold surface using dynamic mechanical deformation. The electrocapillary coupling coefficient in our study, $\Lambda$, measures the response of the capacitive current to the mechanical elastic strain with a focus on the potential range of the double layer capacitive process of interest. The variation in the magnitude of $\Lambda$ during the potential scan shows that the impact of surface strain on the capacitive current is strongly dependent on the electrode states. The positive sign of $\Lambda$ implies that tensile strain can enhance the charging current on the electrode surface, whereas tensile strain has the opposite effect on the discharging current with the potential changing in the negative direction. Moreover, a simple equivalent circuit was used to further characterize the coupling between the capacitive process and mechanical strain at the gold surface for exploring the application in the field of electrochemical energy storage.

(C) The Author(s) 2019. Published by ECS. This is an open access article distributed under the terms of the Creative Commons Attribution 4.0 License (CC BY, http://creativecommons.org/licenses/by/4.0/), which permits unrestricted reuse of the work in any medium, provided the original work is properly cited. [DOI: 10.1149/2.0081912jes]
\end{abstract}

Manuscript submitted March 22, 2019; revised manuscript received June 13, 2019. Published June 27, 2019.

Recent studies have reported on the strong coupling between the surface mechanics and the electrochemistry. The change in the charge state at electrode surface gives rise to surface stress, leading to the volume change of the electrode materials during the electrochemical processes. ${ }^{1-6}$ The mechanical stress/strain can be also used as one of the most effective methods for modulating conventional electrochemical processes. ${ }^{7-18}$ For example, the capacitive process can be directly affected by mechanically stretching the surface of either the crystal metals ${ }^{7}$ or the amorphous materials. ${ }^{8}$ Moreover, the coupling of adsorption enthalpies of the reactants to the surface strain provides a tool for tuning the catalytic activity of electrode materials. ${ }^{9-12}$

The electrode potential, which is normally linked to the adsorption enthalpy status of adsorbates at the electrode surface during the electrosorption processes, was found strongly strain-dependent in previous literature. ${ }^{11,12}$ In the past decades the variation in the electrode potential with respect to the surface strain was theoretically defined and experimentally quantified as a key parameter to investigate the strainmodulated electrochemistry. ${ }^{19-23}$ The metal-electrolyte interface under dynamic strain has been modeled and thoroughly investigated with an equivalent circuit to understand the strain-induced variation of electrode potential in open circuit-conditions. ${ }^{20}$ When the electrochemical circuit is closed for both the capacitive processes and electrocatalysis, the application of mechanical strain to the electrochemical process inevitably gives rise to a current response to the applied strain. ${ }^{17}$ Since the electrode current as one of the key variables reflects the charge kinetics of electrode in electrochemistry, it is an interesting and important topic to monitor and model the coupling between the capacitive current and mechanical strain during the electrochemical capacitive process.

The gold electrodes is taken as an example of investigation of this unexplored area and the mechanical elastic strain was cyclically applied on the surface in present work. While the measurement strategy was characterized by the previously established, the present study addresses the interplay of the dynamics of electro-mechanical coupling with the charging kinetics of metallic electrodes both in experiment and by phenomenological modeling. We further discuss the implications of the observation in present study for the strain response of the charge in the electrochemical capacitive regime. The finding may be of significance in further explorations of the performance enhancement in electrochemical energy storage materials.

\footnotetext{
*Electrochemical Society Member.
}

${ }^{\mathrm{z}}$ E-mail: qibodeng@tjut.edu.cn

\section{Experimental}

The details of the experimental procedures are largely identical to those described in our earlier reports on the electrocapillary coupling at the metal surfaces, see Refs. 7,12. The potential range in this study was controlled within the capacitive charging/discharging process at the strained surface of the gold electrode. All of the experiments carried out in this work used gold thin films $(\sim 50 \mathrm{~nm})$ sputtered onto the polyimide substrate $(\sim 125 \mu \mathrm{m}$, Upilex, UBE) and were investigated in the working electrolyte of $10 \mathrm{mM} \mathrm{H}_{2} \mathrm{SO}_{4}$ (Suprapur, Merck). The electrode potential was controlled using a potentiostat (PG-Stat $302 \mathrm{~N}$ AUTOLAB) and was given relative to the standard hydrogen electrode (SHE). The X-ray rocking curve indicated a strong texture with (111) surface orientation. ${ }^{24}$ The electrode area, $A$, was $\sim 1.2 \pm 0.2 \mathrm{~cm}^{2}$ and was wetted by the electrolyte meniscus in this study. The electrode surface was cyclically strained by an external piezo-actuator system. The strain amplitude was in the order of $10^{-4}$ and the strain frequency was controlled from $0.6 \mathrm{~Hz}$ to $100 \mathrm{~Hz}$ in a sinusoidal waveform. The lock-in amplifier (SR 7270 Signal Recovery) measured the straininduced current modulation from the amplitude of the potential drop over a shunt resistance $(\sim 46 \Omega)$ in series with the CE. Using the sinusoidal strain signal as the reference in lock-in amplifier, the real and imaginary components of the strain-induced current variation were analyzed and detected by the lock-in technique.

\section{Results}

The cyclic voltammogram (CV) of the 111-textured gold electrode (Fig. 1) exhibits the well-known potential range of the nominally capacitive behavior of a metal surface bounded by the onset of oxygen and hydrogen species electrosorption regimes. We now strain the working electrode mechanically by controlling the movement of a piezo-actuator during the potential sweep. Fig. 2a shows the oscillation trace of the capacitive current signal in the time domain as the potential sweeps, while the gold surface is under cyclical strain. We note that the cyan solid curve in Fig. 2a shows the conventional current signal obtained without straining the electrode surface. For illustration, we imposed a constant electrode potential of $0.6 \mathrm{~V}$ and recorded the time-dependence of the applied strain and the current response signal by an oscilloscope. These results are shown in the Fig. 2b. It is readily seen that the current response signal exhibits the same variation frequency as the applied strain. This finding confirms that the current oscillation observed in Fig. 2a is indeed induced by the applied strain, 


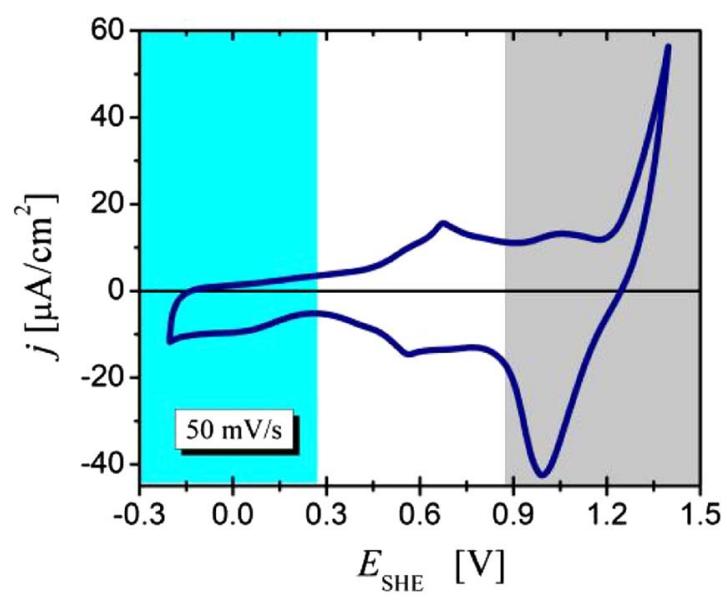

Figure 1. Current density, $j$, plotted versus the electrode potential, $E$ in $10 \mathrm{mM}$ $\mathrm{H}_{2} \mathrm{SO}_{4}$. The scan rate is indicated in the figure.

and the strain-induced current variation is clearly resolved, despite the small amplitude of the applied strain $(\sim 0.02 \%$ in this study).

At first glance, the peak-to-peak amplitude of the capacitive current oscillation observed in Fig. 2a depends on the state of the electrode $(E)$. This cyclic oscillation of the capacitive current induced by a sinusoidal strain signal can either be directly recorded as a part of the voltammogram, or it can be accurately analyzed in terms of the amplitude and the phase using a lock-in amplifier. To describe the extent of the mechanical strain effect, an electrocapillary coefficient of interest is defined as the response of the electrode current to the mechanical strain at the constant potential conditions, ${ }^{7}$ namely, $\Lambda=\left.\frac{\mathrm{d} I}{\mathrm{~d} \varepsilon}\right|_{E}$, where $I$ is the electrode current, $E$ is the electrode potential, and $\varepsilon$ is a tangential strain variable.

Using the first measurement strategy, the peak-to-peak amplitude of the strain-induced current variation was mathematically calculated from the $\mathrm{CV}$ data and is plotted as a function of the electrode potential as shown in Fig. 2c. The lock-in technique was also used to evaluate the strain-induced current variation as the second measurement strategy. The curves obtained by two different strategies agree in the entire potential window. It is observed that the effect of the surface strain on the capacitive current $(|\Delta I / \Delta \varepsilon|)$ depends strongly on the electrode potential, in agreement with the oscilloscopic observation results presented in Fig. 2a. We now discuss the magnitude of $\Lambda$. From the negative limit of the potential, the magnitude value of $\Lambda$ increases in the scan in the positive direction and then reaches a maximum peak value of $+5.3 \pm 0.3 \mathrm{~mA}$ at the potential of $E \approx 0.65 \mathrm{~V}$. Upon further increase in $E$, the coupling coefficient decreases. When the scan direction is inverted, the graph of $\Lambda(E)$ reflects its essentially reversible behavior.

For the first measurement strategy, it is difficult to obtain the phase shift of the current variation relative to the strain signal during the potential sweep due to the large database. Since the lock-in technique uses a sinusoidal strain signal as the reference signal, it can be used to record both the magnitude and the phase shift of the current variation relative to the strain signal in a single measurement during cyclic voltammetry. The phase shift of $\Lambda$ varies somewhat from $20^{\circ}$ to $35^{\circ}$ at the strain frequency of $20 \mathrm{~Hz}$ throughout the entire potential range in our study. The potential interval during cyclic voltammetry was set to around $2 \mathrm{mV}$ when using $10 \mathrm{mV} / \mathrm{s}$ scan rate in our measurement. The equilibrium time at each potential point could be around $200 \mathrm{~ms}$, which is 4 cycles of strain variation $(20 \mathrm{~Hz})$. That is sufficient time to monitor the value of $\Lambda$ by the lock-in amplifier. It can be seen as the effective value of $\Lambda$ at each potential interval. The stable value of $\Lambda$ in the time domain, as shown in Fig. 2d, indicates that the the system is at equilibrium during measurement in Fig. 2c.

Considering that one of the significant features of a capacitive current is that its magnitude depends strongly on the potential scan rate, it is also of interest to determine the relationship between the currentstrain response $(\Lambda)$ and the potential scan rate. The results presented in Fig. 3 probe the behavior of the coupling coefficient $\Lambda$ at the different scan rates of $2 \mathrm{mV} / \mathrm{s}, 5 \mathrm{mV} / \mathrm{s}$ and $10 \mathrm{mV} / \mathrm{s}$. The response values at the positive scan and negative scan show less hysteresis at smaller scan
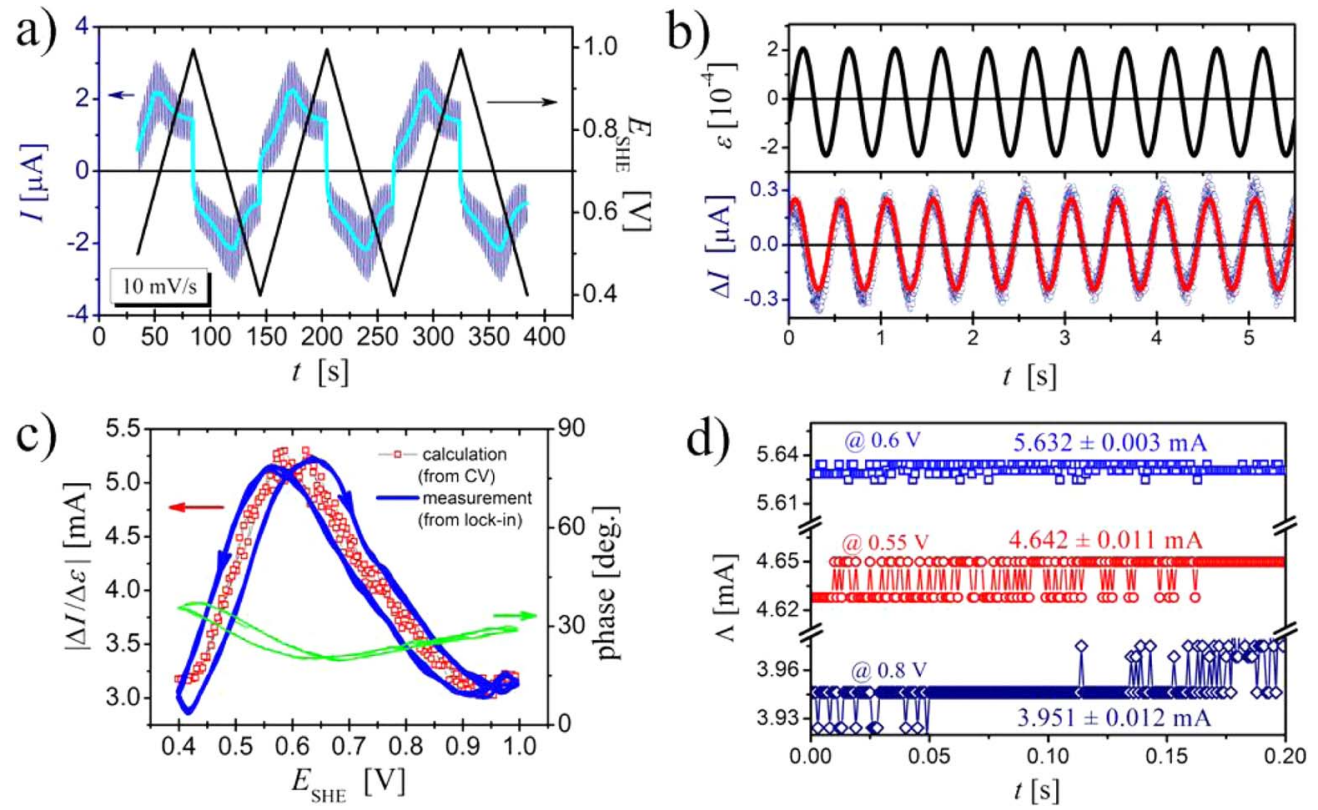

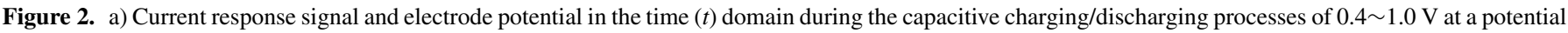

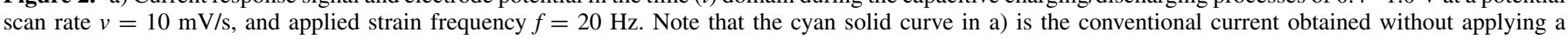

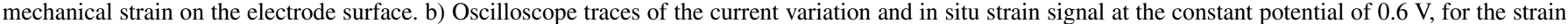

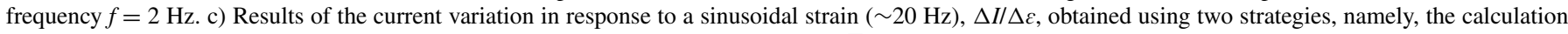

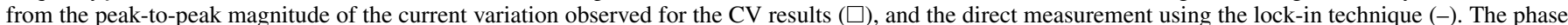

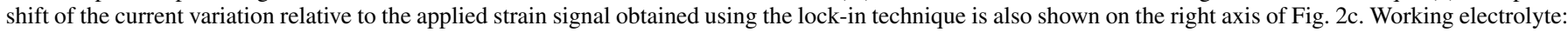
$10 \mathrm{mM} \mathrm{H}_{2} \mathrm{SO}_{4}$. d) The stable value of $\Lambda$ is detected by lock-in amplifier in the time domain at different constant potentials $(f=20 \mathrm{~Hz})$. 

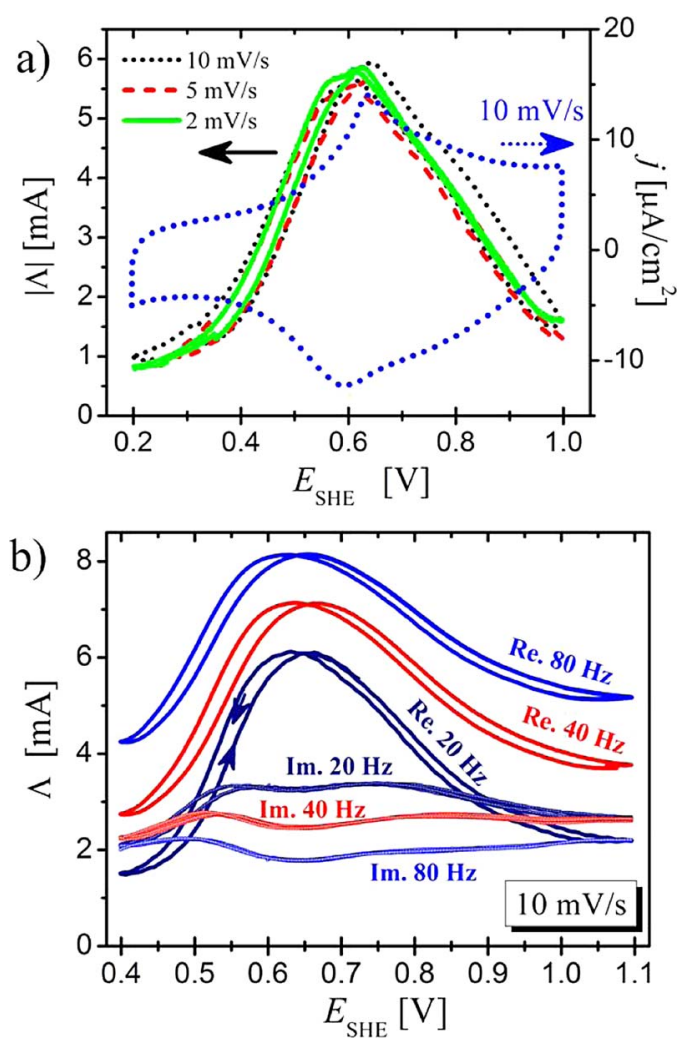

Figure 3. a) Current-strain response $\Lambda(E)$ for Au thin film electrode at the different scan rates of $2 \mathrm{mV} / \mathrm{s}, 5 \mathrm{mV} / \mathrm{s}$ and $10 \mathrm{mV} / \mathrm{s}$. Strain frequency of $20 \mathrm{~Hz}$. b) Real (Re.) and imaginary (Im.) components of $\Lambda(E)$ at different cyclic strain frequencies of $20 \mathrm{~Hz}$ (navy), $40 \mathrm{~Hz}$ (red) and $80 \mathrm{~Hz}$ (blue).

rates, whereas the amplitude of $\Lambda$ is almost independent on the scan rate.

Since a phase shift of the current response signal is related to the applied strain signal (Fig. 2c), the coupling coefficient, $\Lambda$, contains real and imaginary components. We are more interested in the real and imaginary components than on the magnitude and phase because the real and imaginary components can be linked more naturally to the sign inversions. Fig. 3 b shows these real and imaginary components of $\Lambda(E)$ at different strain frequencies of 20,40 and $80 \mathrm{~Hz}$. Throughout the entire potential interval, it is observed that the graphs of $\Lambda(E)$ for different strain states exhibit qualitatively identical features. Nevertheless, the graphs are significantly shifted relative to each other along the ordinate. The real component amplitude increases with increasing strain frequency, whereas the value of imaginary component decreases as the frequency increases. As some of the most important features, the real and imaginary components are systematically set to an identical positive sign within the entire potential range of the capacitive process. The positive value of $\Lambda(E)$ means that a tensile strain can enhance the capacitive current when the potential is swept in the positive direction (charging process), whereas the positive value of $\Lambda$, in the negative direction of the potential sweep, has the opposite physical meaning for restricting the discharging process.

Toward the modeling of the capacitive process at the strained electrode surface, a systematic study on the strain frequency dependence of $\Lambda$ as shown in Fig. 4a and reveals this trend in Fig. 3b to be reproducible when the frequency is scanned from $0.6 \mathrm{~Hz}$ to $100 \mathrm{~Hz}$ at $E=$ $0.6 \mathrm{~V}$. This potential was chosen because it is close to the maximum value of $\Lambda(E)$. Fig. 4a plots the real and imaginary components of the coupling parameter $(\Lambda)$ as a function of the applied strain frequencies. Both real and imaginary components approach zero in the low strain frequency region. The value of the real component increases with increasing strain frequency and reaches saturation above the frequency of $60 \mathrm{~Hz}$. The imaginary component reaches a maximum value close to the strain frequency of $15 \mathrm{~Hz}$. Additionally, the magnitude and the phase shift of $\Lambda$ are plotted in Fig. 4b. It is observed that the magnitude reaches saturation when the frequency of the above $60 \mathrm{~Hz}$ amplitude. The phase shift deceases from $80^{\circ}$ to $10^{\circ}$ with increasing frequency.

\section{Discussion}

In this study, we constructed an equivalent electrical circuit to further understand the current-strain response at the metal-electrolyte interface. The equivalent circuit is shown in the inset of Fig. 4a. In the simplest case, we did not include the losses associated with the charge transfer, and the system behaves as the perfect capacitor because the faradaic processes are excluded and the metal-electrolyte interface charges capacitively:

The excitation is achieved by the time- $(t-)$ dependent strain in a sinusoidal form with an angular frequency $(\omega=2 \pi f), \varepsilon=\hat{\varepsilon} \sin (\omega t)$, where the hatted quantity is the strain amplitude. For the simplest case where the electrode potential reacts immediately to the strain, the potential response is given by: $:^{22}$

$$
E(t)=E_{0}+\varsigma \hat{\varepsilon} \sin (\omega t),
$$

where $\varsigma$ is the potential-strain coupling coefficient defined as $\varsigma=$ $\mathrm{d} E /\left.\mathrm{d} \varepsilon\right|_{q} ^{7,19-22}$ and $E_{0}$ is the conventional variable found without applying the mechanical strain. We then included the source of the elastic strain driven potential variation in the model via $\Delta E(t)=\varsigma \hat{\varepsilon} \sin (\omega t)$. The current modulation by the elastic strain with a phase shift $(\varphi)$ induced by the metal-electrolyte interface can be then written as

$$
\begin{aligned}
I(t) & =I_{0}+|\Lambda| \hat{\varepsilon} \sin (\omega t+\varphi) \\
& =I_{0}+\Lambda_{\mathrm{re}} \hat{\varepsilon} \sin (\omega t)+\Lambda_{\mathrm{im}} \hat{\varepsilon} \cos (\omega t)
\end{aligned}
$$

where $\Lambda_{\mathrm{re}}$ and $\Lambda_{\mathrm{im}}$ are the real and imaginary components, respectively.
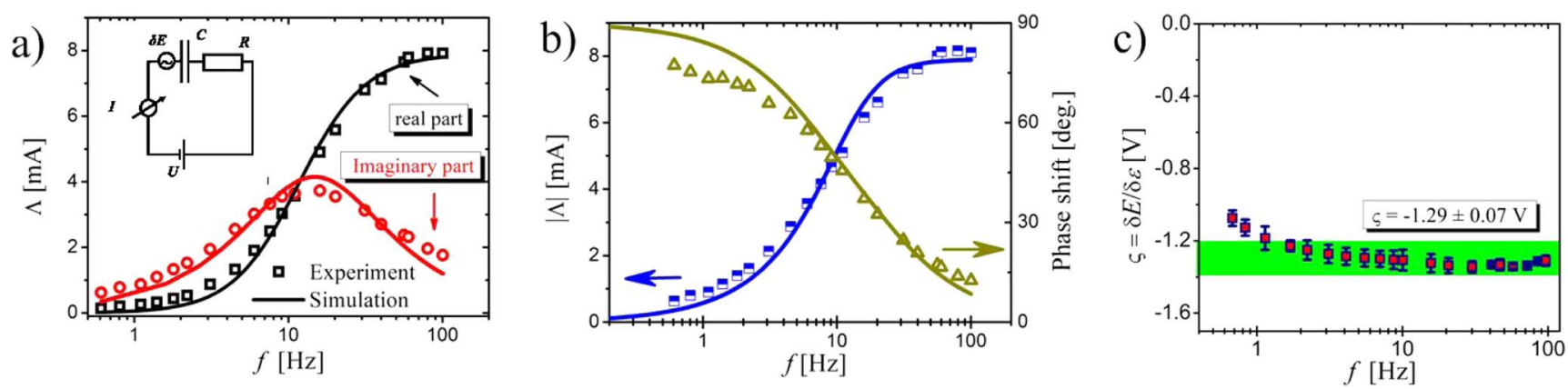

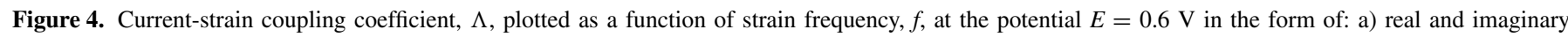

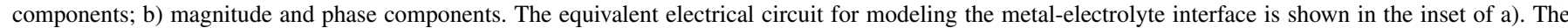

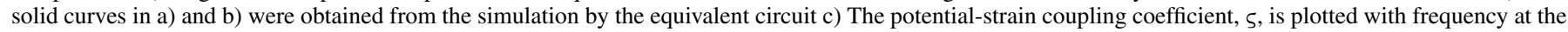
potential $E=0.6 \mathrm{~V}$. Electrolyte: $10 \mathrm{mM} \mathrm{H}_{2} \mathrm{SO}_{4}$. 
The circuit is readily analyzed for the potential variation $\Delta E(t)$ using Kirchhoff's circuit laws. The solution can be parameterized using the coefficient of the potential-strain response $\varsigma$, the capacitance of double layer $C$ and the reciprocal of time constant $\omega_{1}=1 / R C$. The solution of the equivalent electrical circuit for current modulation induced by strain is expressed by

$$
\left\{\begin{array} { l } 
{ \Lambda _ { \mathrm { re } } = - \frac { C \varsigma \omega ^ { 2 } \omega _ { 1 } } { \omega ^ { 2 } + \omega _ { 1 } ^ { 2 } } } \\
{ \Lambda _ { \mathrm { im } } = - \frac { C \varsigma \omega \omega _ { 1 } ^ { 2 } } { \omega ^ { 2 } + \omega _ { 1 } ^ { 2 } } }
\end{array} . \text { Or equivalently, } \left\{\begin{array}{l}
|\Lambda|=\frac{C \varsigma \omega \omega_{1}}{\sqrt{\omega^{2}+\omega_{1}^{2}}} \\
\varphi=\arctan \frac{\omega_{1}}{\omega}
\end{array}\right.\right.
$$

The model was found to be in a good agreement with the experiment at the constant electrode potential, $E=0.6 \mathrm{~V}$. The best fit that is displayed as the solid lines in Figs. $4 \mathrm{a}$ and $4 \mathrm{~b}$ has $\varsigma=-1.24 \pm 0.06 \mathrm{~V}$, $C=86 \pm 2 \mu \mathrm{F}$ and $\omega_{1}=73 \pm 6 \mathrm{~s}^{-1}$. The expression for the imaginary component, $\Lambda_{\mathrm{im}}$, indicates that the maximum response value is $-C_{\varsigma} \omega_{1}$ for $\omega=\omega_{1}$, explaining well the experimental observation of the imaginary part in Fig. 4a.

To further verify the our constructed circuit model, we used the scheme of Dynamic Electro-Chemo-Mechanical Analysis described in our previous reports to obtain the value of $\varsigma$ in this study. ${ }^{7}$ Fig. $4 \mathrm{c}$ represents the dependence of $\varsigma$ on the cyclic strain frequency $(f)$ at the identical conditions in measurement of $\Lambda$ (e.g. $10 \mathrm{mM} \mathrm{H}_{2} \mathrm{SO}_{4}$ electrolyte and the constant potential of $0.6 \mathrm{~V}$ ). Inspection of Fig. $4 \mathrm{c}$ shows that the magnitude of $\varsigma$ increases slightly with $f$, apparently saturating above $1 \mathrm{~Hz}$. This trend is in agreement with previous reports. ${ }^{7}$ The saturated value of $\varsigma$ is approximately $-1.29 \pm 0.07 \mathrm{~V}$. The independent experiment for this value of $\varsigma$ obtained at $E=0.6 \mathrm{~V}$ then supports the correctness of our equivalent circuit model.

We now discuss the implications of this observation for the strain response of the charge in the capacitive regime, which is of interest for the field of electrochemical energy storage. Using a similar definition to that of the current-strain response, the expression for the chargestrain response can be written as

$$
\begin{aligned}
Q & =Q_{0}+\Phi \hat{\varepsilon} \sin (\omega t+\phi) \\
& =Q_{0}+\Phi_{\mathrm{re}} \hat{\varepsilon} \sin (\omega t)+\Phi_{\mathrm{im}} \hat{\varepsilon} \cos (\omega t),
\end{aligned}
$$

where the parameter $\Phi$ is defined as the response of the charge to the applied strain $\left(\Phi=\left.\frac{\mathrm{d} Q}{\mathrm{~d} \varepsilon}\right|_{E}\right)$. Furthermore, based on the well-known relation of current and charge, we can obtain the variation of charge induced by the sinusoidal strain:

$$
\begin{aligned}
Q(t) & =\int I(t) d t=\int\left[I_{0}+\Lambda \hat{\varepsilon} \sin (\omega t+\varphi)\right] d t \\
& =Q_{0}-\frac{\Lambda_{\mathrm{re}} \hat{\varepsilon}}{\omega} \cos (\omega t)+\frac{\Lambda_{\mathrm{im}} \hat{\varepsilon}}{\omega} \sin (\omega t) .
\end{aligned}
$$

Then, we obtain the following mathematical expressions for the charge-strain coupling:

$$
\begin{aligned}
& \left\{\begin{array}{l}
\Phi_{\mathrm{re}}=\frac{\Lambda_{\mathrm{im}}}{\omega}=-\frac{C \varsigma \omega_{1}^{2}}{\omega^{2}+\omega_{1}^{2}} \\
\Phi_{\mathrm{im}}=-\frac{\Lambda_{\mathrm{re}}}{\omega}=\frac{C \varsigma \omega \omega_{1}}{\omega^{2}+\omega_{1}^{2}}
\end{array}\right. \\
& \text { Or equivalently, }\left\{\begin{array}{l}
|\Phi|=\frac{C \varsigma \omega_{1}}{\sqrt{\omega^{2}+\omega_{1}^{2}}} \\
\phi=\arctan \left(-\frac{\omega}{\omega_{1}}\right)
\end{array}\right. \text {. }
\end{aligned}
$$

The charge-strain response calculated from the current-strain response is plotted as a function of the strain frequency in Fig. 5. We obtain the main features of the charge-strain response from the above mathematical expressions of the real and imaginary components of $\Phi$ as follows:

1) Since $\zeta$ is negative during the electrochemical capacitive process, the imaginary component of the charge-strain response, $\Phi_{\mathrm{im}}$, ex-

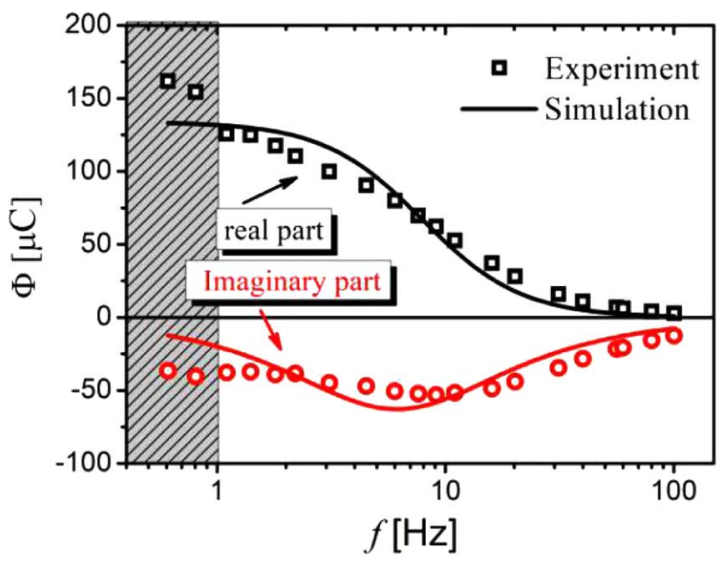

Figure 5. Charge-strain response calculated from the experimental data (scattered points) and the circuit model (solid lines) of the current-strain response in Figure 4a.

hibits a minimum value $0>\Phi_{\text {im }} \geq \frac{C_{\varsigma}}{2}$; and equality is satisfied only when $\omega=\omega_{1}$.

2) When the angular frequency of mechanical strain is considerably higher than the reciprocal of the time constant of the metalelectrolyte interface $\left(\omega \gg \omega_{1}\right)$ :

$$
\left\{\begin{array}{l}
\lim _{\frac{\omega_{1}}{\omega} \rightarrow 0(\omega \rightarrow \infty)} \Phi_{\mathrm{re}}=0 \\
\lim _{\frac{\omega_{1}}{\omega} \rightarrow 0(\omega \rightarrow \infty)} \Phi_{\mathrm{im}}=0 .
\end{array} .\right.
$$

3) When the angular frequency of mechanical strain is much lower than the reciprocal of the time constant of the metal-electrolyte interface $\left(\omega \ll \omega_{1}\right)$ :

$$
\left\{\begin{array}{l}
\lim _{\frac{\omega}{\omega_{1}} \rightarrow 0(\omega \rightarrow 0)} \Phi_{\mathrm{re}}=-C \varsigma \\
\lim _{\frac{\omega}{\omega_{1}} \rightarrow 0(\omega \rightarrow 0)} \Phi_{\mathrm{im}}=0
\end{array} .\right.
$$

It is observed that the charge at the electrode surface is indeed enhanced by tensile static strain where the strain frequency could be considered to be zero because the coupling coefficient $\Phi$ has the same positive sign as the conventional charge. These data imply that by controlling the external strain with a mechanical method on the electrode surface, one can obtain a faster charging rate or slower discharging rate covering the most important range of the electric double layer.

\section{Conclusions}

Let us summarize our argument: the coupling between the capacitive current and mechanical strain that is a significant factor in the metal-electrolyte interface during the surface charging/discharging process can be measured by the mechanical deformation of solid bodies when charged in the electrolyte. Due to the presence of capacitor behavior at the metal-electrolyte interface, the strain-induced current variation depends strongly on the applied strain frequency and also depends on the electrode potential. A simple equivalent circuit was used to explain the special interface where the electrode surface is strained. The key finding in this study is that the tensile strain can enhance the capacitive current while charging the electrode surface, whereas the tensile strain has the opposite effect to inhibit the discharging process. This finding implies that the charging rate can be adjusted by the mechanical method. Based on the experimental results and the model analysis, the relationship between the surface charge and the applied strain was explored in order to study and understand the strain-dependence of the electrochemical processes. By controlling the surface strain states, e.g., tensile or compressive amplitude, the conventional electrochemical charging/discharging processes can be further enhanced. 


\section{Acknowledgments}

Q. Deng thanks Prof. Jörg Weissmüller for the helpful discussions. This study was supported by the National Natural Science Foundation of China (No. 11602171) and was partly funded by Deutsche Forschungsgemeinschaft, grant WE1424/16-1. Q. Deng is also grateful for the support from the "Hundred Talents Program" of Tianjin University of Technology. A critical reading by Xinyan Wu is gratefully acknowledged.

\section{ORCID}

Qibo Deng (I) https://orcid.org/0000-0003-3383-9358

\section{References}

1. W. Haiss, Surface stress of clean and adsorbate-covered solids, Rep. Prog. Phys., 64, 591 (2001).

2. J. Weissmüller, R. N. Viswanath, D. Kramer, P. Zimmer, R. Würschum, and H. Gleiter, Charge-induced reversible strain in a metal, Science, 300, 312 (2003).

3. J. W. Shin, U. Bertocci, and G. R. Stafford, In situ stress measurement during hydrogen sorption on utrathin (111)-textured Pd films in alkaline electrolyte, J. Electrochem. Soc., 158, F127 (2011).

4. K. Wang, C. Stenner, and J. Weissmüller, A nanoporous gold-polypyrrole hybrid nanomaterial for actuation, Sensor. Actuat. B-Chem., 248, 622 (2017).

5. M. C. Lafouresse, U. Bertocci, C. R. Beauchamp, and G. R. Stafford, Simultaneous electrochemical and mechanical impedance spectroscopy using cantilever curvature, J. Electrochem. Soc., 159, H816 (2012).

6. H. Wang, X. Shan, H. Yu, Y. Wang, W. Schmickler, H. Chen, and N. Tao, Determining electrochemical surface stress of single nanowires, Angew. Chem. Int. Ed., 129, 2164 (2017)

7. M. Smetanin, Q. Deng, and J. Weissmüller, Dynamic electro-chemo-mechanical analysis during cyclic voltammetry, Phys. Chem. Chem. Phys., 13, 17313 (2011).

8. H. Zhang, C. An, A. Yuan, Q. Deng, and J. Ning, A non-conventional way to modulate the capacitive process on carbon cloth by mechanical stretching, Electrochemistry Communications, 89, 43 (2018).
9. L. A. Kibler, A. M. El-Aziz, R. Hoyer, and D. M. Kolb, Tuning reaction rates by lateral strain in a palladium monolayer. Angew. Chem. Int. Ed., 44, 2080 (2005).

10. P. Strasser, S. Koh, T. Anniyev, J. Greeley, K. More, C. Yu, Z. Liu, S. Kaya, D. Nordlund, H. Ogasawara, M. F. Toney, and A. Nilsson, Lattice-strain control of the activity in dealloyed core-shell fuel cell catalysts, Nat. Chem., 2, 454 (2010).

11. J. He, Y. Shen, M. Yang, H. Zhang, Q. Deng, and Y. Ding, The effect of surface strain on the CO-poisoned surface of Pt electrode for hydrogen adsorption, J. Catl., 350, 212 (2017).

12. Q. Deng, M. Smetanin, and J. Weissmüller, Mechanical modulation of reaction rates in electrocatalysis, J. Catal., 309, 351 (2014).

13. M. Luo and S. Guo, Strain-controlled electrocatalysis on multimetallic nanomaterials, Nature Reviews Materials, 2, 17059 (2017).

14. A. Khorshidi, J. Violet, J. Hashemi, and A. A. Peterson, How strain can break the scaling relations of catalysis, Nature Catalysis, 1, 263 (2018).

15. H. Wang et al., Direct and continuous strain control of catalysts with tunable battery electrode materials. Science, 354, 1031 (2016).

16. Q. Deng, V. Gopal, and J. Weissmüller, Less noble or more noble: how strain affects the binding of oxygen on gold, Angew. Chem. Int. Ed., 54, 12981 (2015).

17. K. Yan, T. A. Maark, A. Khorshidi, V. A. Sethuraman, A. A. Peterson, and P. R. Guduru, The influence of elastic strain on catalytic activity in the hydrogen evolution reaction, Angew. Chem. Int. Ed., 55, 6175 (2016).

18. J. Weissmüller, R. N. Viswanath, L. A. Kibler, and D. M. Kolb, Impact of surface mechanics on the reactivity of electrodes, Phys. Chem. Chem. Phys., 13, 2114 (2011).

19. G. Valincius, Elastic electrocapillary properties of polycrystalline gold, Langmuir, 14, 6307 (1998)

20. M. Smetanin, D. Kramer, S. Mohanan, U. Herr, and J. Weissmüller, Response of the potential of a gold electrode to elastic strain, Phys. Chem. Chem. Phys., 11, 9008 (2009).

21. J. Weissmüller and D. Kramer, Balance of force at curved solid metal-liquid electrolyte interfaces, Langmuir, 21, 4592 (2005).

22. Q. Deng and J. Weissmüller, Electrocapillary coupling during electrosorption, Langmuir, 30, 10522 (2014).

23. C. Stenner, L. Shao, N. Mameka, and J. Weissmüller, Piezoelectric gold: strong charge-load response in a metal-based hybrid nanomaterial, Adv. Funct. Mater, 26, 5174 (2016).

24. Q. Deng, D.-H. Gosslar, M. Smetanin, and J. Weissmüller, Electrocapillary coupling at rough surfaces, Phys. Chem. Chem. Phys., 17, 11725 (2015). 